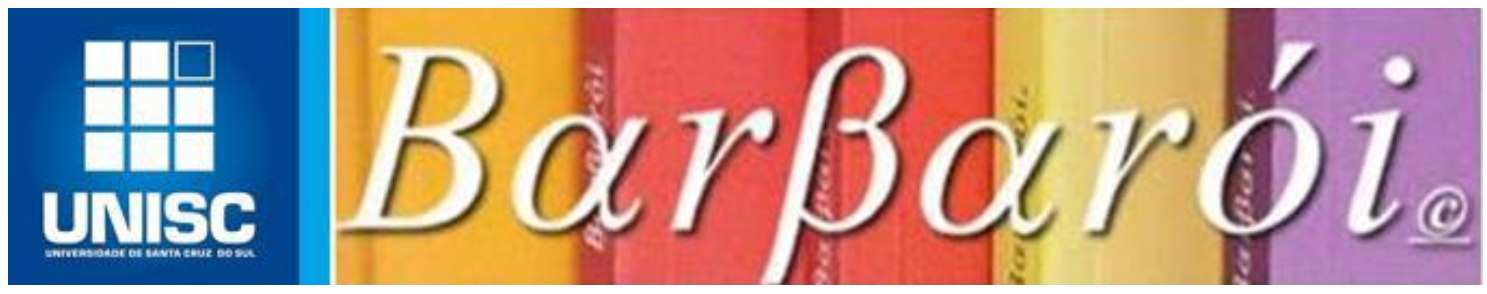

\title{
POLÍTICA DE SAÚDE MENTAL NO BRASIL: ALGUNS APONTAMENTOS
}

DOI: http://dx.doi.org/10.17058/barbaroi.v1i53.13289

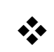 \\ Ellayne Karoline Bezerra da Silva \\ Universidade Federal do Piaui - UFPI - Brasil
}

\section{Resumo}

Estudo sobre a trajetória das políticas sociais no Brasil com recorte ao período de 1930 aos dias atuais, utilizando o método de pesquisa bibliográfica, de modo a mostrar mais especificamente o resgate histórico da política de saúde mental brasileira enquanto política de saúde, identificando suas principais características e os desafios que permeiam esta política no contexto social atual. De acordo com os autores estudados, percebeu-se que o sistema de proteção social brasileiro, mesmo diante dos significativos progressos quanto a ampliação dos direitos sociais, ainda é definido como um sistema de não redistributividade, reflexo da sua construção que foi caracterizada pela centralização política e financeira no governo federal e nas ações sociais, fragmentação institucional, exclusão da população à participação política, o autofinaciamento social, a privatização e o clientelismo que ainda persiste em muitos segmentos sociais. Reflexos estes que também perpassam pelas demais políticas públicas, incluindo a de saúde, bem como saúde mental, que está envolta por grandes desafios na sua trajetória de construção.

Palavras chaves: Políticas sociais. Política de saúde. Política de saúde mental.

\section{Introdução}

Pretende-se com esse trabalho fazer uma abordagem analítica acerca da política de saúde mental enquanto política de saúde integrada às políticas sociais. Assim, é de grande relevância entender o desenvolvimento, organização e características do sistema de proteção social no Brasil, tendo em vista que seus reflexos serão direcionados para todas as políticas.

Inicialmente mencionam-se alguns aspectos quanto à trajetória das políticas sociais brasileiras, tratando em seguida acerca do percurso histórico de constituição e consolidação da assistência psiquiátrica no Brasil, destacando os avanços e retrocessos desta trajetória, bem como seus marcos legais. 
Descreve ainda um breve histórico da loucura e das políticas brasileiras com o intuito de expor as principais modificações na área da saúde mental, pois se acredita que só assim se compreenda o contexto pelo qual perpassa o modelo psiquiátrico atual, em processo de substituição. Nesse contexto, é tratado da Reforma Psiquiátrica e dos Centros de Atenção Psicossociais, como um dos dispositivos fundamentais desse processo.

\section{Os desdobramentos da política social brasileira: algumas considerações}

Inicialmente este trabalho analisa a trajetória das políticas sociais brasileiras com recorte ao período de 1930 aos dias atuais, pautado nas discussões trazidas por Draibe (1990), Melo (1993) e Arrethc (2002), de forma a situar o desenvolvimento dessas políticas, bem como as suas nuances.

A análise de Draibe (1990) fundamenta-se no sistema de proteção social, Welfare State, enquanto "uma particular forma de regulação social que se expressa pela transformação das relações entre o Estado e a economia, entre o Estado e a sociedade, a um dado momento do desenvolvimento" (DRAIBE, 1988 apud DRAIBE, 1990, p. 02).

Assim, o sistema de proteção social brasileiro define-se em três etapas bem delimitadas por Draibe (1990): a primeira fase, de 1930 a 1943, caracterizada pela criação dos Institutos de Aposentadorias e Pensões - IAPs, pela regulamentação relativa das leis trabalhistas, bem como pelos avanços significativos nas políticas de saúde e educação com nacionalização da política e centralização do governo federal; a segunda fase, de 1945 a 1964, com a expansão do sistema de proteção social, com um viés seletivo, heterogêneo e fragmentado da intervenção estatal; e a terceira fase, que parte de meados da década de 60 a meados da década de 70, que significou a fase de consolidação do sistema de proteção social, marcado por um quadro radical de transformação da armação institucional e financeira do perfil da política social, caracterizado pelos acelerados processos de industrialização, urbanização e transformação da estrutura social.

Segundo a autora, somente nos anos 70, as tendências universalizantes foram sendo introduzidas no sistema através dos IAPs, incorporações de outras categorias na previdência, domésticas e rurais, autônomos, expansão do ensino básico gratuito, criação do Sistema Nacional de Habitação. Em contrapartida, essas tendências reforçaram o caráter meritocrático do sistema de proteção social frente ao seu caráter redistributivo.

Historicamente, portanto, o sistema de proteção social brasileiro está envolvo no binômio igualdade/universalidade X meritocracia/seletividade. Dessa forma, Draibe (1990) caracteriza o Estado de Bem Estar Brasileiro basicamente sobre o princípio do mérito, “entendido basicamente como a posição ocupacional e de renda adquirida ao nível da estrutura Barbarói, Santa Cruz do Sul, n.53, p.<172-184>,jan./jun. 2019 
produtiva, que constitui a base a qual se ergue o sistema brasileiro de política social" (DRAIBE, 1990, p. 09-10).

Para Draibe (1990, p. 15), a consolidação do Estado de Bem Estar Brasileiro está baseado nos seguintes princípios:

\section{[...] extrema centralização política e financeira no nível federal das ações sociais do Governo; acentuada fragmentação institucional; exclusão da participação social e política dos processos decisórios; o principio do autofinanciamento do investimento social; o principio da privatização; o uso clientelístico da máquina social (DRAIBE, 1990, p. 15).}

O contexto brasileiro nos anos 80 caracteriza-se pela abertura do sistema, transição para a Nova República e pela Constituição de 1988, que refletiram no aumento e redirecionamento do gasto social, reforma no perfil da proteção social e da reforma da máquina administrativa do Estado. Para Melo (1993, p. 141) "um período inicial de esforço concentrado, seguido de fragmentação e descontinuidades".

Embora nesse período as políticas sociais tenham ampliado os direitos sociais através da "universalização do acesso e a expansão da cobertura, um certo afrouxamento do vínculo contributivo como princípio estruturante do sistema" (DRAIBE, 1990, p. 29), o sistema de políticas sociais ainda remonta a concepção de "dar mais a quem tem menos" (DRAIBE, 1990, p. 33), mostrando o caráter celetista, focalizado, concentrado em alguns objetivos e nos segmentos mais necessitados da população.

Assim, as características das políticas sociais assumem uma verdadeira negação de Estado Protetor, tendo em vista que são corporativistas, reguladoras, fragmentadas, focalizadas, controladoras de uma integração seletiva, heterogênea e autoritária, com normas e padrões desiguais de proteção. Políticas direcionadas para uma pequena parcela da população reconhecida legalmente pelo Estado, em detrimento de uma grande maioria excluída, hierarquizada, discriminada e marginalizada como classe trabalhadora pobre. Para essa grande maioria prevalecia à negação de direitos.

Já em meados da década de 90, a configuração das políticas sociais brasileiras apresentava tendências descentralizadas em várias áreas (especificamente nas áreas de saúde, educação, assistência social e habitação), porém se deu de forma diferenciada, por variações de diversas ordens: "quanto ao momento no tempo em que deu início ao processo de descentralização de cada política social; quanto à extensão (ou 'sucesso') da efetiva transferência de funções de cada política de um nível de governo para outro" (ARRETCH, 2002, p. 34). 
Desse modo, fez-se de grande importância mencionar sucintamente a trajetória das políticas sociais no Brasil para entendermos como estas se desenvolvem para suprir as velhas e novas demandas sociais, bem como os desdobramentos deste histórico nas demais políticas, principalmente na Política de Saúde e especificadamente de Saúde Mental.

\section{TRAJETÓRIA DA ASSISTÊNCIA PSIQUIÁTRICA NO BRASIL}

\section{Assistência Psiquiátrica no Brasil}

Inicialmente os considerados "loucos" eram institucionalizados na Santa Casa de Misericórdia, que foram os primeiros serviços brasileiros de assistência hospitalar, vinculados às irmandades religiosas. (ROSA, 2008)

Nesse período, os loucos quando calmos e tranquilos eram tratados juntos a sociedade, mas se agitados e inquietos, eram aprisionados em cadeias públicas. A questão da loucura também se atrelava a outros determinantes sociais, já que os loucos que faziam parte de famílias ricas eram tratados no próprio domicílio ou enviados para tratamento na Europa, enquanto os pobres ficavam nas ruas, cadeias ou mesmo sob cuidados de caridade. (ROSA, 2008)

A assistência psiquiátrica no Brasil surge em meados do século XIX, com forte influência europeia. Em 1852, é inaugurado o primeiro hospício no Brasil localizado no Rio de Janeiro com a denominação de Hospício Pedro II, marcando o modelo que viria a ser chamado de hospitalocêntrico. Nesse período o hospício surge como um meio de sanear e proteger a sociedade, visto que os loucos eram considerados perigosos e, portanto um "caso de polícia". (ROSA, 2008)

Com a chegada do período republicano, a assistência psiquiátrica no Brasil ganha o aspecto científico, e junto a isso há a desvinculação do Hospício Pedro II da Santa Casa de Misericórdia, rompendo com a visão caritativa e assistencialista, passando a designar-se Hospício Nacional dos Alienados.

Nesse período, houve algumas experiências com o intuito de inovar o modelo psiquiátrico, uma delas foi às colônias agrícolas que objetivava "fazer a comunidade e os loucos conviverem fraternalmente, em casa ou no trabalho" (AMARANTE 1994 apud LIMA, 2008, p. 14). A proposta das colônias agrícolas abrangia um tratamento extra-asilar, baseado em atividades do trabalho rural, proporcionando ou pelo menos tendo como finalidade proporcionar liberdade aos loucos. Proposta esta que não obteve êxito e foi alvo de duras críticas, por que representava um retrocesso diante de uma sociedade que estava em desenvolvimento industrial e urbano. Em outras palavras, os loucos eram adaptados a uma realidade diferente das mudanças enfrentadas pela sociedade brasileira.

Barbarói, Santa Cruz do Sul, n.53, p.<172-184>,jan./jun. 2019 
Nesse contexto, com a criação da Liga Brasileira de Higiene Mental (LBHM), em 1923, no Rio de Janeiro surge o Movimento de Higiene Mental. A LBHM tinha caráter marcadamente “eugenista, antiliberal, moralista e xenofóbico" (COSTA, 1980 apud ROSA, 2008, p. 91). Acreditava-se que os fatores sociais poderiam ser controlados de tal forma que conseguiriam alterar as raças, tanto no seu aspecto físico quanto mental, além de apoiar-se na profilaxia para responder as questões de saúde mental.

Na década de 60, mais especificamente com a ditadura militar no Brasil, a saúde mental transforma-se num verdadeiro objeto de lucro, tendo em vista a privatização da assistência psiquiátrica pelo Estado. Assim, é criado o Instituto Nacional de Previdência Social (INPS), o qual estendia a assistência psiquiátrica a "todo trabalhador e seus dependentes, segurados no sistema" (ROSA, 2008, p. 99), reforçando o que foi chamado de "indústria da loucura", por financiar leitos em serviços privados e ampliar o rol de enfermidades mentais, como o alcoolismo.

Em contraponto a "indústria da loucura", é criada a Divisão Nacional de Saúde Mental (DINSAM), setor responsabilizado pela "normatização, avaliação e controle dos programas de saúde mental” (ROSA, 2008, p. 101), com características preventivas, pautado na ambulatorização da assistência psiquiátrica e na redução de internações. Entretanto ainda persiste o grande investimento nos leitos hospitalares privados, com financiamentos públicos através dos convênios.

Nesse mesmo período, surge o Movimento de Reforma Sanitária, que se baseava no fortalecimento da sociedade civil e na construção de um sistema de saúde universal e de responsabilidade estatal. O Movimento de Reforma Sanitária propôs a "transformação do sistema de saúde vigente por um sistema democrático, universal, acessível e socialmente equitativo" (OLIVEIRA, MARTINHAGO, MORAES, 2009, p. 20). Esses objetivos foram alcançados com a Constituição Federal de 1988, que deu origem ao Sistema Único de Saúde (SUS) e define no seu Art. 196 a saúde como:

[...] direito de todos e dever do Estado, garantido mediante políticas sociais e econômicas que visem à redução do risco de doença e de outros agravos e ao acesso universal e igualitário às ações e serviços para sua promoção, proteção e recuperação (BRASIL, 1988, p.127).

Concomitante ao Movimento de Reforma Sanitária e a crise da DINSAM, explicada pelas recorrentes denúncias de trabalhadores de saúde metal em relação aos tratamentos desumanos e precários dos serviços prestados, surge o Movimento dos Trabalhadores em Saúde Mental (MTSM) e o Movimento da Reforma Psiquiátrica. 
Assim, o MTSM tem caráter contestador promovendo denúncias, referentes à "violação de direitos humanos dos portadores de transtorno mental; abordagem da loucura como fonte de lucro e condições precárias de trabalho em hospitais psiquiátricos" (ROSA, 2008, p. 105) e dissemina, portanto, a necessidade de uma reforma na assistência psiquiátrica. E é nessa conjuntura que o Movimento da Reforma Psiquiátrica ganha visibilidade.

O Movimento de Reforma Psiquiátrica no Brasil é influenciado pela tradição basagliana, e emerge em contraposição ao modelo hospitalocêntrico, pautado somente na internação e medicalização como meio de cura, buscando o tratamento baseado nos vários âmbitos da vida do sujeito e "promovendo integração cotidiana entre a loucura e a sociedade". (OLIVEIRA, MARTINHAGO, MORAES, 2009, p.07).

Com a VIII Conferência Nacional de Saúde, a Reforma Psiquiátrica alcança avanços significativos, visto que a saúde, como já explicitado, é assumida como responsabilidade do Estado e seu conceito é ampliado. Além de promover o desenvolvimento da I Conferência Nacional de Saúde Mental, em 1987 , no Rio de Janeiro, com o intuito de fazer a saúde mental acompanhar os avanços da saúde em geral.

De acordo com Rosa (2008), essa Conferência trouxe como principais propostas:

- A reafirmação dos princípios da participação da sociedade civil no processo decisório.

- A substituição do modelo hospitalocêntrico por serviços extra-hospitalares, pautado no trabalho com equipes multiprofissionais.

- A extinção dos leitos psiquiátricos asilares em substituição por leitos psiquiátricos em hospitais gerais públicos, ou mesmo outros serviços, como hospital-dia, hospital-noite e lares protegidos.

- A retomada dos princípios democráticos quanto à realização dos concursos públicos.

- A devolução dos direitos das pessoas com transtorno mental no que se refere a sua cidadania plena.

Tais propostas obtiveram materialidade com a criação e discussões propiciadas pelo projeto de lei $n^{\circ} 3657 / 89$ do mineiro e Deputado Federal Paulo Delgado, que defendia a "extinção dos manicômios e sua substituição por serviços extra-hospitalares" (ROSA, 2008, p. 114). Essa proposta foi alvo de duras críticas segundo a argumentação de que a extinção dos hospitais psiquiátricos asilares conduziria a total desassistência às pessoas com transtorno mental, responsabilizando somente os familiares nessa atividade.

Entretanto, o projeto de lei não propunha essa desassistência, mas o tratamento além dos sintomas e baseado nas particularidades de cada sujeito, diante dos serviços que evitassem a internação, mantendo a pessoa com transtorno mental no convívio comunitário.

Barbarói, Santa Cruz do Sul, n.53, p.<172-184>,jan./jun. 2019 
Nos anos 90, com a intervenção do ideário neoliberal, são protelados os princípios conquistados com a Constituição de 1988 devido à concepção política do governo nas ações seletivas e focalizadas. Mesmo nesse cenário, é realizado, em 1992, a II Conferência Nacional de Saúde Mental com o tema “A reestruturação da atenção em saúde mental no Brasil: modelo assistencial/ direito à cidadania, reafirmando as propostas da VIII Conferência Nacional de Saúde e os princípios do SUS”. Frente a tal realização acontece em 1995 o II Encontro do Movimento de Luta Antimanicomial e no ano seguinte, a X Conferência Nacional de Saúde.

A partir daí, a rede de atenção à saúde mental se expande com os dispositivos substitutivos aos hospitais psiquiátricos, advindos dos princípios da Reforma Psiquiátrica: os Núcleos de Atenção Psicossocial (NAPS), que mais tarde passaram a ser denominados de Centros de Atenção Psicossocial (CAPS); Serviços de Residências Terapêuticas (SRT), Programa de Volta para Casa e a redução dos leitos psiquiátricos. O primeiro CAPS foi criado em 1986, localizava-se em São Paulo e recebeu a denominação de Professor Luiz da Rocha Cerqueira, ou como ficou mais conhecido CAPS Itapeva. (BRASIL, 2004).

É importante frisar que a proposta de Lei Paulo Delgado só foi aprovada depois de doze anos que tramitava no Congresso Nacional, e em outra versão que aniquilou do projeto original a extinção dos manicômios no país, sendo conhecida, portanto como Lei Federal no 10.216/01.

Desse modo, a Reforma Psiquiátrica critica o modelo biomédico, por estar centralizado somente nos sintomas do sujeito e não conceber a pessoa com transtorno mental na sua totalidade e complexidade.

Entretanto, o Movimento da Reforma Psiquiátrica não é homogêneo, compartilhando de várias tendências distintas.

\footnotetext{
Uma que radicaliza a luta pela extinção da estrutura asilar/ manicomial, tornando-se intransigente ao entender que é impossível trabalhar, defender e assegurar direitos dos portadores de transtorno mental no interior destas instituições. Acredita que é só a partir dos espaços extra-hospitalares que pode ser criada uma nova cidadania para o portador de transtorno mental. Uma segunda postula a inserção nos espaços asilares e, a partir deles, defende a implementação de mudanças no modelo assistencial (ROSA, 2008, p. 105).
}

Em geral, a Reforma Psiquiátrica Brasileira está inserida em um contexto social e político de lutas para a garantia da cidadania da pessoa com transtorno mental e contra as práticas de violência nos manicômios, o que é consenso entre as diferentes tendências que disputam a hegemonia, direção do processo.

Mas, observa-se que a partir do novo milênio, a ala conservadora da psiquiatria ganha novo impulso e passa a lutar contra a Reforma Psiquiátrica, a fim de garantir a centralidade nesse processo. 


\title{
A Reforma Psiquiátrica Brasileira
}

A Política de Saúde Mental, desde a década de 90, toma rumos distintos, tendo em vista o novo modelo de atenção à pessoa com transtorno mental, advindos com os ideais da Reforma Psiquiátrica e com a manifestação da Luta Antimanicomial, que visam à substituição do modelo hospitalocêntrico, centrado nas internações e medicalização da doença, devendo ser priorizado o sujeito em sua totalidade, o contexto social em que está inserido, sendo necessário captar os determinantes sociais, econômicos e culturais circundantes à pessoa com transtorno mental (PCTM).

Dessa forma, o novo modelo de assistência às pessoas com transtorno mental ganha maior visibilidade com a aprovação da Lei n ${ }^{\circ}$ 10.216, de 06 de abril de 2001, também conhecida como Lei da Reforma Psiquiátrica ou do deputado Paulo Delgado, visto que teve sua autoria, que redireciona a atenção a saúde mental para uma rede de base comunitária e dispõe sobre os direitos das pessoas com transtorno mental.

\begin{abstract}
Ela garante ser de responsabilidade do Estado o desenvolvimento da política de saúde mental, a assistência e a promoção de ações de saúde aos portadores de transtornos mentais, com a devida participação da sociedade e da família, a qual será prestada em estabelecimento de saúde mental, assim entendidas as instituições ou unidades que ofereçam assistência em saúde aos portadores de transtornos mentais. Esta preconiza que o tratamento visará, como finalidade permanente, a reinserção social do paciente em seu meio, e a internação, em qualquer de suas modalidades, só será indicada quando os recursos extra-hospitalares se mostrarem insuficientes (VELOSO \& CARLOS, 2009, p. 104).
\end{abstract}

Assim, a Reforma Psiquiátrica tem como propostas: 1) Processo de desinstitucionalização, 2) A redução da internação hospitalar em manicômios e 3) Rede de Atenção à Saúde Mental.

O processo de desinstitucionalização caracteriza-se por implicar novos contextos de vida para as pessoas com transtorno mental, bem como para seus familiares e "pretende mobilizar como atores os sujeitos sociais envolvidos, modificar as relações de poder entre os usuários e as instituições e produzir diversas ações de saúde mental substitutivas à internação no hospital psiquiátrico". (OLIVEIRA, MARTINHAGO, MORAES, 2009, p.33)

É interessante mencionar que a desinstitucionalização não pode ser confundida com a desospitalização, visto que a proposta da Reforma não se resume a retirada dos usuários de hospitais, envolvendo um cenário mais amplo caracterizado pela extinção do preconceito e maus-tratos existentes dentro das instituições, além da compreensão do sujeito na sua totalidade e não mais centrado na doença.

De acordo com Oliveira, Martinhago e Moraes (2009) baseado na Declaração de Caracas (1990), o processo de redução da internação hospitalar nos manicômios é explicado Barbarói, Santa Cruz do Sul, n.53, p.<172-184>, jan./jun. 2019 
pela necessidade de transformação das ações como, o isolamento das pessoas com transtorno mental da sociedade e dos principais meios de convivência; a captação de recursos financeiros elevados, sem ao menos proporcionar condições humanas e físicas para os usuários; e também por ser um modelo que não respeita as reais necessidades da saúde mental.

Para que houvesse a redução da internação hospitalar nos manicômios, foi proposta a organização da Rede de Atenção à Saúde Mental, sendo esta conceituada como "uma teia que engloba todos os serviços de saúde, mecanismo que pode promover autonomia e cidadania das pessoas com transtornos mentais" (OLIVEIRA, MARTINHAGO, MORAES, 2009, p.39).

Nesse contexto contribuíram para tal organização as portarias que juntamente com a Lei $\mathrm{n}^{\circ} 10.216$ instituiu as experiências institucionais como os Centros de Atenção Psicossocial (CAPS), Serviços de Residências Terapêuticas (SRT) e o Programa de Volta para Casa.

Os CAPS - Centros de Atenção Psicossocial, atualmente regulamentados pela Portaria $n^{\circ} 336 / G M$ de 19 de fevereiro de 2002, é destinado a acolher as pessoas com transtornos mentais severos e persistentes, de forma a inseri-las na vida comunitária e familiar, buscando assim, a sua autonomia. Funcionam como porta de entrada aos serviços para ações relacionadas à saúde mental, sendo um articulador com outras redes que oferecem serviços a este público, como o Programa de Saúde da Família, ambulatórios, Residências Terapêuticas, entre outros.

O Serviço Residencial Terapêutico (SRT) ou Residências Terapêuticas, de acordo com Brasil (2004) são casas localizadas no espaço urbano, constituídas para responder às necessidades de moradia de pessoas com transtornos mentais graves, institucionalizadas ou não. Assim, são direcionadas as pessoas que estão internadas há um período significativo em hospitais psiquiátricos e não possuem vínculos familiares ou comunitários que garantam "espaço adequado de moradia".

Outro dispositivo de substituição ao antigo modelo psiquiátrico é o Programa de Volta para Casa que foi instituído pela Lei $\mathrm{n}^{\circ} 10.708$, de 31 de julho de 2003 e regulamentada pela portaria $n^{0}$ 2077/2003, que apregoa a assistência e acompanhamento das pessoas com transtornos mentais fora da unidade hospitalar e que já passaram por longo período de internação psiquiátrica equivalente a dois ou mais anos.

Percebe-se ainda a implementação do Programa Nacional de Avaliação do Sistema Hospitalar/Psiquiatria (PNASH/Psiquiatria) e o Programa Anual de Reestruturação da Assistência Hospitalar Psiquiátrica no SUS (PRH), ambos tem como finalidade a redução dos leitos psiquiátricos e a consequente expansão do novo modelo de assistência baseado na rede comunitária. 
Vale ressaltar que a Rede de Atenção à Saúde Mental não se reduz as instituições substitutivas aos hospitais psiquiátricos, envolve desde as já mencionadas instituições, incluindo também os vínculos familiares, as associações e comunidades que de forma compartilhada contribuem para o cuidado e reinserção das pessoas com transtorno mental no convívio social.

Desse modo, o novo cenário da política voltada para as pessoas com transtornos mentais é marcado pela transição gradual do modelo hospitalocêntrico para o modelo proposto pela Reforma Psiquiátrica, onde ainda verificam-se práticas do modelo médico, herança da longa tradição manicomial presente no modo como as PCTM foram e são tratadas, convivendo com práticas orientadas para a reabilitação psicossocial, ampliando os laços sociais das PCTM.

\section{O Centro de Atenção Psicossocial como principal dispositivo reformista}

A proposta da Reforma Psiquiátrica está direcionada a um tratamento mais humano diante do transtorno mental, buscando meios que possibilitem às pessoas com esta enfermidade, inserção social e cidadania, limitando a internação e a exclusão social, visto que esses antigos mecanismos que ainda estão presentes na sociedade são ineficientes no tratamento e na reabilitação psicossocial.

Assim, os CAPS (Centros de Atenção Psicossocial) surgem como um dos dispositivos substitutivos ao modelo hospitalocêntrico, pauta-se no tratamento voltado para reabilitação psicossocial para fortalecer esses sujeitos no convívio familiar e social, promovendo assim um retorno a atividades cotidianas de lazer e trabalho.

O CAPS representa, portanto, um dos modelos alternativos, mas não o único, devendo estar em inteira conexão com o mundo social, ou seja, a referida instituição deve potencializar a desinstitucionalização dos usuários dos serviços de saúde mental, no sentido da contraposição às instituições fechadas, totalitárias e aprisionadoras das subjetividades das pessoas com transtornos mentais.

Nesse contexto e de forma a efetivar o seu papel de provisão das necessidades humanas à PCTM, o CAPS atende preferencialmente pessoas com transtornos mentais severos e persistentes, visando diminuir o número de internações e garantir o convívio social.

O primeiro CAPS do Brasil foi inaugurado em 1986, no Estado de São Paulo, sendo denominado como "Centro de Atenção Psicossocial Professor Luiz da Rocha Cerqueira”, mais conhecido como CAPS da Rua Itapeva. Esse CAPS, assim como os demais são resultantes dos vários movimentos sociais, tendo destaque para o Movimento dos Trabalhadores de Saúde Mental, que buscavam o progresso da assistência psiquiátrica no Brasil. 
Integrados na rede do SUS e regulamentados pela Portaria n ${ }^{\circ} 336 / G M$ de 19 de fevereiro de 2002, os CAPS, conforme BRASIL (2004) objetivam:

- Prestar atendimento em regime de atenção diária;

- Gerenciar os projetos terapêuticos oferecendo cuidado clínico eficiente e personalizado;

- Promover a inserção social dos usuários através de ações intersetoriais que envolvam educação, trabalho, esporte, cultura e lazer, montando estratégias conjuntas de enfrentamento dos problemas;

- Dar suporte e supervisionar a atenção à saúde mental na rede básica, PSF (Programa de Saúde da Família), PACS (Programa de Agentes Comunitários de Saúde);

- Regular a porta de entrada da rede de assistência em saúde mental de sua área;

- Coordenar junto com o gestor local as atividades de supervisão de unidades hospitalares psiquiátricas que atuem no seu território;

- Manter atualizada a listagem dos pacientes de sua região que utilizam medicamentos para a saúde mental.

O CAPS divide-se em três modalidades de acordo com o grau de complexidade e o número de habitantes do município (CAPS I, CAPS II, CAPS III), existindo CAPS específico para o atendimento de crianças e adolescentes (CAPSi) e para usuário de álcool e outras drogas (CAPS ad).

O CAPS I é instalado em municípios com a população entre 20.000 e 70.000 habitantes; o CAPS II entre 70.000 e 200.000; o CAPS III acima de 200.000; o CAPSi também acima de 200.000 habitantes, só que é direcionada para crianças e adolescentes que possuem transtornos mentais; e o CAPSad para municípios com população acima de 100.000 habitantes para pessoas que sofrem com o uso abusivo de álcool e outras drogas.

O CAPS oferece tratamento dividido em três níveis: intensivo, na qual o usuário permanece na instituição em dois turnos durante todos os dias úteis da semana; semi-intensivos, três vezes na semana no período da manhã ou tarde; e não-intensivo, que fica apenas um dos turnos, uma vez por semana.

O tratamento oferecido pelo CAPS é acompanhado por uma equipe multiprofissional composta por assistentes sociais, psicólogos, psiquiatras, enfermeiros, terapeutas ocupacionais, auxiliares de enfermagem, técnicos administrativos, dentre outros.

Portanto, os CAPS representam o que há de mais avançado no que diz respeito ao processo de reabilitação psicossocial das pessoas com transtornos mentais, mas tendo em vista que do ponto histórico são muito recentes e tem como principal tarefa a formação de uma nova 
cultura, para além dos manicômios, com o objetivo primeiro na condução de uma sociedade que não estigmatize o diferente, e possibilite a convivência familiar e comunitária das PCTM.

\title{
Considerações finais
}

O sistema de proteção social brasileiro teve grandes conquistas significativas na ampliação dos direitos sociais, no entanto, ainda é definido como um sistema não redistributivo, resultante da sua construção que foi caracterizado pela centralização política e financeira no governo federal e nas ações sociais, fragmentação institucional, exclusão da população à participação política, o autofinaciamento social, a privatização e o clientelismo que ainda persiste em muitos segmentos sociais.

Aspectos estes que contribuem para o desenvolvimento das demais políticas, mais precisamente direcionou-se este trabalho às políticas de saúde e diretamente à política de saúde mental, que teve sua construção permeada pelos reflexos das características da política social e do seu desenvolvimento.

Logo, a Política de Saúde Mental está envolvida por grandes desafios na sua trajetória, sendo que as propostas de reforma dessa política encontra-se em um processo lento, que traz avanços e recuos diante das diferentes forças que se apresentam em sua conjuntura, bem como do sucateamento das políticas sociais através dos ideários neoliberais que também afetam o campo da saúde mental.

\section{MENTAL HEALTH POLICY IN BRAZIL: SOME NOTES}

\begin{abstract}
Study on the trajectory of social policies in Brazil with cut between 1930 to the present day, based on literature review, in order to show more specifically historical review of mental health policy while Brazilian health policy, identifying its main features and challenges that permeate this policy in the current social context. According to the authors studied, it was realized that the Brazilian social protection system, despite the significant progress and the expansion of social rights, is still defined as a system of redistribution is not a reflection of its construction has been characterized by political centralization financial and in the federal government and in social, institutional fragmentation, exclusion of the population to political participation, self financing social, privatization and patronage that still persists in many segments of society. These reflections which also permeated by other public policies, including mental health, which is surrounded by large challenges in the construction of the precepts that come with the Psychiatric Reform.
\end{abstract}

Keywords: social policy. Health policy. Mental health. 


\section{Referências}

Arretch, Marta. Estado federativo e políticas sociais: determinantes da descentralização. São Paulo: Revan, 2002.

BRASIL. Constituição (1988). Constituição da República Federativa do Brasil. Brasília, DF: Senado Federal: Centro Gráfico, 1988.

Ministério da Saúde. Secretaria de Atenção à Saúde. Departamento de Ações Programáticas Estratégicas. Saúde Mental no SUS: os Centros de Atenção Psicossocial Brasília: Ministério da Saúde, 2004.

Ministério da Saúde. Secretaria de Atenção à Saúde. Lei n10. 216, de 6 de abril de 2001.Dispõe sobre a proteção e os direitos das pessoas portadoras de transtornos mentais e redireciona o modelo assistencial em saúde mental. fevereiro de 2002.

Ministério da Saúde. Secretaria de Atenção à Saúde. Portaria 336/GM de 19 de

DRAIBE, S. As políticas sociais brasileiras: diagnósticos e perspectivas para a década de 90. In: IPEA, Brasília: IPEA, 1990.

LIMA, Leopoldina Coelho. Serviço social no CAPS Leste: a prática na perspectiva da reabilitação psicossocial. Trabalho de conclusão de curso, UFPI. Teresina: 2008.

MELO, M. A. Autonomia do fracasso: intermediação de interesses e a reforma das políticas sociais na Nova República. Revista de Ciências Sociais, n. 1 v. 36, 1993.

OLIVEIRA, Walter Ferreira de; MARTINHAGO, Fernanda; MORAES, Ramona Sant Ana Maggi de. Entendendo o processo da reforma psiquiátrica: a construção da rede de atenção à saúde mental. Florianópolis: ABRASME/UFSC, 2009.

ROSA, Lúcia Cristina dos Santos. Transtorno mental e o cuidado na família. $2^{\circ}$ ed. São Paulo: Cortez, 2008.

VELOSO, Lorena U.P.; CARLOS, Karolyna P. T. A nova política de saúde mental no Estado do Piauí: mudanças e perspectivas. IN: ROSA, Lúcia Cristina dos Santos; Guimarães, Lucas Dannilo Aragão; CARVALHO, Marta Evelin Bona de (orgs.). Cenários de práticas em saúde mental: a atenção psicossocial no Piauí. Teresina: EDUFPI, 2009.

Data de recebimento: 09/03/2019

Data de aceite: 17/09/2019

\section{Sobre a autora:}

Ellayne Karoline Bezerra da Silva é graduada em Serviço Social pela Universidade Federal do Piauí (2010), Mestre em Políticas Públicas pela Universidade Federal do Piauí (2015) e Doutora em Políticas Públicas pela Universidade Federal do Piauí (2019). Atua principalmente nos seguintes temas: transtorno mental, saúde mental, família e políticas públicas. Endereço Eletrônico: layne.karoline@ hotmail.com 